\title{
Prescolarisation Et Developpement Des Apprentissages Fondamentaux A L'ecole Primaire Ivoirienne. Une Analyse Comparee D'enfants De Milieux Differents
}

\author{
Seka Yapi Arsène Thierry \\ École Normale Supérieure d’Abidjan département \\ des Sciences de l’Éducation
}

doi: 10.19044/esj.2016.v12n28p329 URL:http://dx.doi.org/10.19044/esj.2016.v12n28p329

\begin{abstract}
Pre schooling is an institutionalized period of pre learning where child coordinates his sense, to adjust his actions to reach to read and write in teaching language; French. But although an unequal repartition of schools garden, and mostly not having attended these schools, some children reach to read and write as well as possible in French language. This research gave the occasion to compare oral and written productions of children according their status (pre scolarised and not pre scolarised). The aim is to evaluate the two children group's performance on oral and written knowing that one group live in town so has attended garden school and the other group live in campaign where there is no garden. The hypothesis according which, there is a bound between attending garden and developing of children's performance shows that there is a deep difference between the two groups of children. The one who attended garden school are talented in reading whereas they who are in campaigns succeed more in writing.
\end{abstract}

Keywords: School garden, Learning, Reading, Writing

\section{Resume}

La préscolarisation est une période de préapprentissage institutionnalisée où l'enfant coordonne ses sens, ajuste ses actions pour parvenir à lire et écrire dans la langue d'enseignement ; le français. Mais malgré l'inégale répartition des écoles maternelles, et sans les avoir fréquentées pour la plupart des cas, des enfants parviennent à lire et écrire tant bien que mal en langue française. Cette étude a permis de comparer les productions orales et écrites du français, des enfants selon leur statut (préscolarisés et non préscolarisés). L’objectif étant d'évaluer les performances à l'oral et à l'écrit des deux groupes d'enfants sous-entendu 
que l'un est préscolarisé et l'autre non. L’hypothèse du lien entre la fréquentation de la maternelle et le développement des performances des enfants aboutit au résultat selon lequel il existe une différence significative entre les deux groupes d'enfants. Les enfants préscolarisés sont doués à l'expression orale tandis que ceux des campagnes le sont plus en écriture.

Mots Clés : Préscolarisation, Apprentissage, Lecture, Écriture

\section{Introduction}

Parler d’apprentissage scolaire dans le primaire préparatoire, c’est essentiellement s'interroger sur le langage à travers ses deux fonctions que sont l'oral et l'écrit. La caractéristique du premier cycle de l'école primaire est l'apprentissage de la lecture et de l'écriture à partir d'une langue d'enseignement qui est le français dans le contexte ivoirien. La connaissance du français dès l’école maternelle constitue un accélérateur au développement des fonctions psychologiques chez les jeunes apprenants. Aussi, importe-il d'emmener l'enfant à développer la langue et à l'intérioriser pour qu'elle lui serve de «médiateur, d'outil pour accéder à un mode de pensée abstraite ». Bruner (1983 : 9).

Mais le rôle de la préscolarisation comme vecteur de développement des fonctions cognitives de l'enfant pourrait être controversé dans la mesure où l'école maternelle n'est pas présente partout en Côte d'Ivoire. La complexité de la situation met également en présence de jeunes apprenants dont les compétences linguistiques sont aussi variées qu’opposées. D’un côté, certains apprenants, par ailleurs les plus nombreux, évoluent dans un contexte où ils n’ont pas bénéficié de préscolarisation et où la langue d'accès à l'expression de leurs expériences quotidienne est différente de la langue d'enseignement.

De l’autre côté, la présence d’enfants préscolarisés et apparemment doués à l'utilisation orale de la langue d'enseignement, présume d'un développement sans accrochage de leurs compétences scolaires. Deux groupes d'enfants de milieux différents et de langues parfois différentes, soumis dans les mêmes conditions d'apprentissage. Notre intérêt pour cette étude se justifie par le caractère hybride que présente le contexte. Notre objectif vise à évaluer les compétences dans le développement du langage oral et écrit chez les apprenants avec l'hypothèse d'un lien entre la préscolarisation et le développement des apprentissages scolaires chez l'enfant. Pour y parvenir une analyse contextuelle de l'école maternelle semble déterminante pour la suite de notre démarche. 


\section{I/ contexte de l'etude}

En Côte d'Ivoire, l'école maternelle en tant qu'institution, date de 1958. La première école maternelle a vu le jour à Bouaké (centre de la côte d'ivoire) pour s’étendre 7 ans plus tard dans d'autres villes comme Abidjan et Daloa. De 1965 à 1968, 52 écoles ont vu le jour dont 27 écoles publiques et 25 dans le privé. On note une régression dans les effectifs. En effet, de 2877 élèves en 1965, l’on est descendu à 2425 élèves en 1968. A partir de 1970, on enregistre une progression surtout dans le privé. Au niveau de l'enseignement public, des études ont montré que de 1970 à 1977, l'école maternelle n’a pas évolué, elle a plutôt connu une stagnation au profit de l'école primaire avec l'avènement de l'enseignement télévisuel.

L’enseignement télévisuel, une autre méthode pédagogique est une copie du modèle canadien. Cette méthode pédagogique voulue par les autorités ivoiriennes répondait aux aspirations d'une Côte d'Ivoire dotée d'un système éducatif à la dimension des grandes nations américaines. Dès le début des années 70, des agents sont envoyés au Canada pour une formation technique et pédagogique du nouvel outil didactique. Le nouveau programme télévisuel s’étend sur toute l'étendue du territoire national, la ville de Bouaké abrite le centre émetteur du programme de montage et de diffusion des émissions. La population adhère à la nouvelle méthode d'enseignement à cause de l'aspect distractif et novateur qu'est la télévision.

L’État de son côté, ne ressent plus le besoin de créer des écoles maternelles puisque tous les enfants en âge de scolarisation sont inscrits directement à l'école télévisuelle. Le préscolaire n’attirait plus parce qu'on y voyait aucun intérêt surtout dans les zones rurales. Tout semble réussir jusqu'au début des années 80. La côte d’Ivoire est secouée par une crise économique ; le poids des dettes extérieures oblige les institutions de breton Wood à la soumettre à un plan d'ajustement structurel. Désormais, les ressources financières sont faibles et ne permettent plus de faire face à l'importation et à l'entretien du matériel télévisuel.

Les stages de recyclage des techniciens au Canada sont supprimés. L’État ne peut plus assurer la maintenance des appareils; toutes choses qui conduisent par voie de conséquence, à l'abandon de l'enseignement télévisuel. Le besoin de rattraper la création des écoles maternelles resurgit dès lors, mais les moyens financiers ne peuvent suivre. Le privé prend le relais dans les grandes villes, mais la grande majorité des enfants issus des zones rurales sont malheureusement abandonnés à leur sort.

L'école maternelle publique représente environ 54,25\% de l'effectif global avec $48,85 \%$ de filles et $51,15 \%$ de garçons. L'écart est insignifiant. Le district d'Abidjan présente un déséquilibre dans les infrastructures du préscolaire ; cela est perceptible dans les communes. A titre d'exemple, l'inspection de l'enseignement primaire de Cocody 2 compte 14 écoles 
publiques contre seulement 02 dans la commune d'Abobo où il y a plus de 1.000.000 habitants. Il y a moins d’écoles maternelles publiques là où la population est importante. Comparativement à la zone rurale, on constate que le préscolaire est essentiellement un phénomène urbain, à en croire les chiffres dans le tableau ci-dessous.

Tableau 1 : répartition des écoles maternelles selon l'habitat en Côte d'Ivoire zone rurale zone urbaine

$\begin{array}{lllr}\text { Effectif } & 297 & 41592 & \text { total } \\ \text { Pourcentage } & 1,4 \% & 91889 \\ & 1,6 \% & 100 \%\end{array}$

Source : Ministère de l'éducation nationale, (2010). Rapport final sur l'évaluation des structures du préscolaire. Abidjan : Auteur.

Il y a plus d'enfants scolarisés en zone urbaine qu'il y en a en zone rurale qui pourtant totalise une population de 8000000 d'habitants avec $70 \%$ d'analphabètes. L'effectif du préscolaire 41592 fournis par la DIPES ${ }^{13}$ ne représente que 6,2\% de la population des enfants de 3 à 6 ans, en âge d'aller à l'école. La majorité 93,8\% des enfants ne bénéficient pas du préscolaire en Côte d'Ivoire. L'enseignement préscolaire connaît une très mauvaise répartition. Sur l'ensemble du territoire, on observe que c'est un phénomène exclusivement urbain qui s'explique non pas par un souci de mode de garde mais à une réelle demande de socialisation et de confrontation aux apprentissages en vue de réussir sa scolarité. Bautier (2006). Les établissements préscolaires sont concentrés dans les villes, conférant ainsi aux zones urbaines, des atouts réels susceptibles de favoriser le développement des compétences scolaires des enfants. C'est dans un tel contexte que nous tenterons de mener une étude comparative sur les performances de la langue orale et écrite des élèves en CP1. Il est entendu ceux des élèves qui sont en ville ont tous fait la maternelle contrairement à ceux des zones rurales qui n’ont pas ce privilège.

\section{Methodologie}

La méthodologie repose essentiellement sur une démarche expérimentale. Partir de la formulation d'une problématique pour arriver au recueil des données sur le terrain, en passant par la thèse et les hypothèses à vérifier, ne renvoie pas systématiquement à une démarche purement expérimentale. Cependant, tout porte à croire que, ce que nous avons à réaliser s'inscrit bien dans une approche expérimentale. Dans la forme, il s’agira de déterminer les relations de causalité entre variables indépendantes et variables dépendantes.

${ }^{13}$ DIPES : Direction des Stratégies de la Planification et des Statistiques. 
Objectif général : L’étude vise à analyser les difficultés graphiques des apprenants des classes de CP1.

\section{Objectifs spécifiques}

- $\quad$ Identifier les facteurs d'explication des difficultés d'écriture.

- $\quad$ Évaluer l'effet de ces facteurs sur les séances d'écriture.

Hypothèse générale : Les difficultés d'apprentissage de l'écriture sont occasionnées par les facteurs liés l’environnement scolaire et culturel.

\section{Hypothèses spécifiques}

- Il existe un lien entre la préscolarisation et les performances en écriture

- Un lien peut également être établi entre la maturité physique et le succès en écriture.

\section{Taille et composition de l'échantillon}

L'échantillon actif est composé de 135 sujets répartis comme suit : 60 sujets à Adzopé à l'EPP Habitat et 75 autres à l'EPP Nyan. L'enquête se fait à partir de deux localités distinctes. La ville d'Adzopé est un département situé dans le sud Est de la Côte d'Ivoire où toutes les fonctions de l'administration centrale sont représentées. Nyan est un village situé à $20 \mathrm{Km}$ d'Adzopé. Les seuls agents de l'État sont les instituteurs. IL n’y a pas d'école maternelle. La population est paysanne et l'agriculture manuelle reste l'activité principale.

Tableau 2 : Répartition des élèves selon le milieu et la préscolarisation.

$\begin{array}{ccc}\begin{array}{c}\text { Tableau 2: Répartition des élèves selon le milieu et la préscolarisation. } \\ \text { Préscolarisés } \\ \text { Cp1 } \mathrm{A}=30\end{array} & \begin{array}{l}\text { Non préscolarisés } \\ \text { EPP Habitat }\end{array} \\ \begin{array}{c}\mathrm{Cp} 1 \mathrm{~B}=30 \\ \text { EPP Nyan }\end{array} & & \mathrm{Cp} 1 \mathrm{~A}=35 \\ \text { Total } & \mathbf{6 0} & \mathrm{Cp} 1 \mathrm{~B}=40 \\ & & 75\end{array}$

135

\section{Instrument de recueil des données}

Le principe est d'évaluer chez l'enfant, ses compétences en écriture. L’écriture cursive constitue l'unité de mesure. L’évaluation est faite sur la note de 10 . Les enfants ayant une note dans l'intervalle de 5 à 10 sont classés dans la catégorie des bons élèves en écriture. Deux épreuves sont prévues pour l'évaluation; les exercices sont les suivants;

1/ Expression écrite :

Complète avec un mot que tu connais: Maman porte un ... / Mariam joue à la ... 
2/ Copie : René va à l'école.

\section{Outil et Méthode d'analyse des données}

L’observation est centrée sur les élèves en situation réelle d'apprentissage pour évaluer leur niveau de maîtrise de la lecture. Nous pouvons également observer à travers la même séance, le comportement de l'élève et son niveau d'adaptation dans son nouvel environnement scolaire. Elle se construira sur la base des résultats chiffrés assortis de l'effet des variables déjà indiquées.

\section{Traitement des données}

SPSS (statistical package for social sciences) est le logiciel de traitement de données en vue d'analyses statistiques. Le traitement est fait sur la base des données issues de l'observation. Elle concerne les performances des élèves aux différentes épreuves de lecture et d'écriture. Nous avons d'abord procédé à l'inscription des variables indépendantes dans les cellules de tableaux ; ensuite, nous avons enregistré successivement les variables dépendantes que sont les différentes performances aux différentes épreuves subies. A ces performances, nous avons procédé à un système de codage : le chiffre 1 est affecté aux enfants qui réussissent aux épreuves et le chiffre 0 affecté à ceux des enfants qui ne réussissent pas l'épreuve. Ce système a été valable tant pour les épreuves de lecture que d'écriture. Enfin, après avoir enregistré toutes ces données, nous lançons l'opération et obtenons les résultats statistiques, (les khi-deux, les mesures, tableaux, et les graphiques, que nous commentons dans une série d'analyses.

\section{Resultats}

Tableau 3 : habitat et Performance des sujets à l'épreuve du recopiage.

$\begin{array}{ccc} & \text { Oui } & \text { non } \\ \text { Zone urbaine } & 52 & 8 \\ \mathrm{~N}=60 & 86,67 \% & 13,33 \% \\ \text { Zone rurale } & 56 & 19 \\ \mathrm{~N}=75 & 74,67 \% & 25,35 \% \\ \text { Total } & 108 & 27 \\ \mathrm{~N}=135 & 80 \% & 20 \%\end{array}$


Diagramme 1 de la répartition des sujets à l'épreuve d'écriture selon l’habitat.

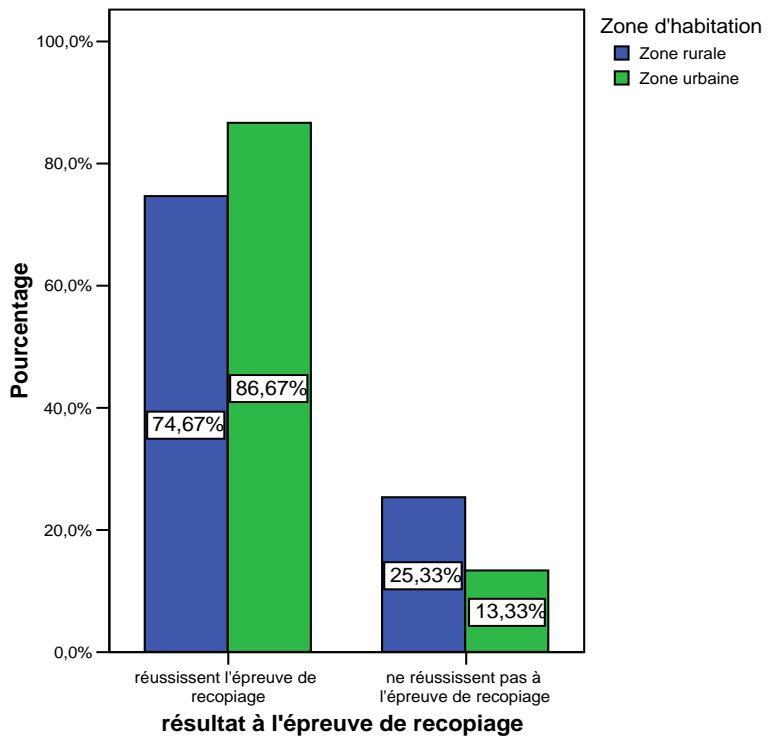

L’analyse du tableau illustrée par le graphique ci-dessous, montre des différences de performance. Chez les enfants des zones urbaines, 52 succès enregistrés soit (86,67\%) de réussite et (13,33\%) d’échec. En zone rurale, 56 enfants réussissent soit $(74,67 \%)$ de réussite et $(25,33 \%)$ d’échec. Une lecture approfondie permet de dire que, en termes de proportion, les enfants en zones rurales (les plus nombreux) réussissent mieux que ceux des zones urbaines. Le khi-deux n'est pas significatif au seuil de 5\% car le khi-deux expérimental $(3,000)$ est inférieur au khi-deux théorique $(3,841)$.

Mais notons qu'au seuil de $10 \%$, le test est significatif car la valeur du khi-deux tabulé correspondant est $(2,706)$.

Quelques productions écrites d’élèves selon l'habitat et l'âge.

Figure 1.Zone rurale. 9ans

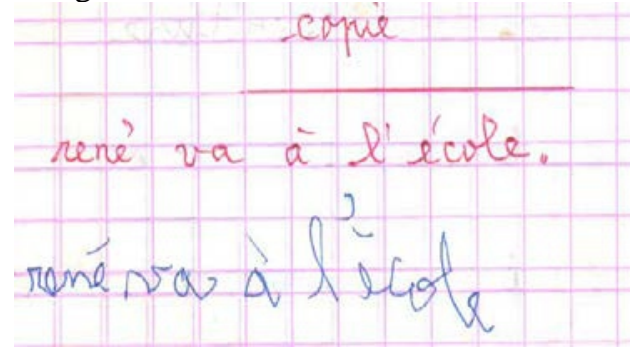

Figure 2. Zone urbaine. 5ans

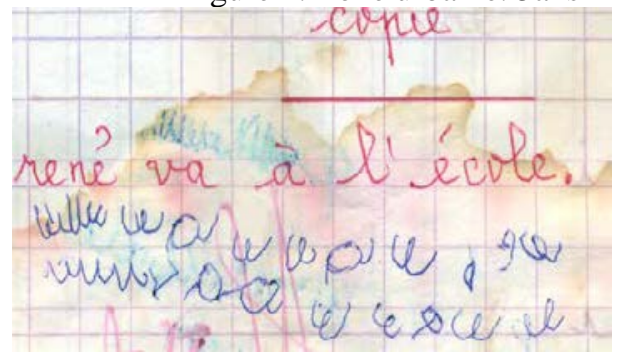


Figure 3. Zone rurale. 9ans

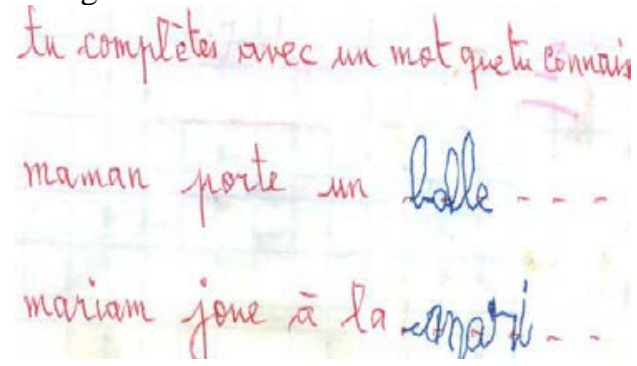

Figure 4. Zone urbaine. 5ans

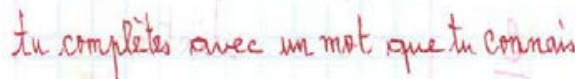

maman ponte uns-ovese-

mariam jone à la.

Les figures 1 et 3 représentent les performances d'un élève de de 9ans de la zone rurale, tandis que les 2 et 4 correspondent à celles d'un élève de 5ans déjà préscolarisé. Il vit en zone urbaine. La différence graphique entre les deux élèves peut s'expliquer par le facteur âge avec tous les attributs qui y sont rattachés entre autres, le développement de la psychomotricité. Par ailleurs, si on note une bonne performance en écriture chez l'enfant de campagne, ses performances en expression écrites restent très faibles comme en témoigne la figure 3. La langue d'enseignement peut être pour lui, un ralentisseur de ses apprentissages scolaires.

\section{Discussion}

\section{Préscolarisation : un stimulant à l'écriture}

Afin de répondre aux objectifs du ministère de l'éducation nationale et de la formation de base, une organisation pédagogique structurée autour de trois sections a été mise en place au préscolaire: petite section, moyenne section, grande section. Le programme en vigueur tourne autour des activités suivantes : langage, graphisme, exercices sensoriels, activités manuelles, motricité, chant, conte, poésies. A ces activités s'ajoutent celles des mathématiques dans les moyennes et grandes sections. Malgré la grande ressemblance des intitulés, les contenus sont de difficultés croissantes. En $\mathrm{CP} 1$, l'unanimité autour des performances que réalisent les enfants préscolarisés à l'échelle d'évaluation des compétences, préalables aux apprentissages fondamentaux par rapport aux enfants non préscolarisés, n'est pas véritablement contestée.

Cette différence traduit l'existence d'un lien significatif entre la préscolarisation et le niveau d'acquisition des enfants dans toutes les épreuves sauf en écriture. Pour comprendre le succès général des enfants préscolarisés, une compréhension des avantages de la préscolarisation est nécessaire. Pour Sauvage (2003), l'école maternelle est un lieu de socialisation qui assure chez l'enfant l'éveil, favorisant du coup une certaine élasticité de son expression langagière qui lui assure une bonne oralité pour entrer plus tard, dans l'écrit. En raison de la dynamique interactionnelle qu'elle favorise et du développement des capacités cognitives qu'elle suscite 
chez l'enfant, Passerieux (2009), admet que plus tôt l'enfant y entre, davantage ses apprentissages se consolideront. Dans le processus d'apprentissage cognitif, la théorie de l'interaction sociale met en évidence l'importance de la langue et de sa compréhension dans la construction des opérations mentales et des connaissances. Dans le développement des capacités cognitives, Vygostky (1985) prend en compte la dimension sociale.

Le langage structure la pensée et la pensée développe le langage. L'enfant, par le langage, a la possibilité d'exprimer un choix doublé d'appréciation. Les divers types d'activités (contes, jeux dramatiques, manipulations, représentations ou relations...) sollicitent l'engagement total de l'enfant ; ce qui suppose une mise en œuvre, similaire du mouvement, de l'expression, du rythme et du langage. L'importance du préscolaire se traduit de façon significative dans les productions linguistiques de l'enfant. La lecture de certains résultats renseignent sur la capacité des enfants à réaliser, dans des proportions objectives, les objectifs fixés par le préscolaire pour faciliter le développement des capacités de l'enfant sur les plans socio affectif, psychomoteur et cognitif. En effet, l'intégration de l'enfant au sein d'une nouvelle société à vivre avec d'autres enfants, à être responsable et autonome, lui fait découvrir des valeurs de référence. Il acquiert ainsi des comportements, des attitudes qui lui permettent d'être imprégné de sa culture. A travers le jeu, l'enfant découvre le besoin de communiquer, il éprouve du plaisir à échanger avec les autres. Non seulement l'enfant développe son langage en interaction avec le groupe, mais fortifie par la même occasion, par l'activité physique, ses muscles et nerfs pour ajuster sa latéralité.

A travers l'apprentissage de la langue, des opérations logiques et des représentations spatio temporelles à la maternelle, l'enfant accroît rapidement ses structures cognitives. L'apprentissage de la langue d'enseignement dès l'école maternelle, constitue le meilleur pronostic de la réussite dans les autres disciplines. L'approche de solution au problème de la langue au cours préparatoire réside dans la mise en place du système d'enseignement à l'école maternelle. Bernicot (2009) s'inspire de Vygotski à qui il fait référence dans son ouvrage pour soutenir qu'il existe en chacun un langage intérieur qui joue un rôle fondamental dans l'exercice des opérations mentales et dans l'acquisition des connaissances. Si on demande à un enfant d'entourer dans un ensemble de formes (carré, rond, triangle...), des ronds bleus ; pour l'approche de solution, l'enfant engage une série d'opérations mentales. Il effectue une analyse dans un langage intérieur dont l'effet apparaît dans les réponses ou dans les résultats de son action. Le point focal de la pensée de Vygostky identifie le langage comme outil de développement de la pensée. 
En effet, l'interaction entre l'enfant et l'objet d'une part et entre l'enfant et l'éducatrice préscolaire d'autre part, provoque des échanges. Cette phase d'activité du langage permet à l'enfant de développer des compétences linguistiques et par conséquent améliorer ses performances scolaires. L'apprentissage du langage est pour l'enfant du préscolaire, un moment d'excitation orale et écrite que l'école maternelle explore en s'appuyant sur des situations d'échange les plus usuels et les plus pratiques. Grace à la compréhension de la langue d'enseignement, l'enfant évoque des faits éloignés, rend présent l'objet absent, désigne, nomme, identifie ainsi, il accède à la représentation symbolique qui pour Piaget, (2006) constitue un ensemble d'instruments cognitifs qui permettent à l'enfant d'être capable d'évoquer des situations ou des objets absents.

L’école maternelle doit servir de tremplin à développer le langage pour fonder les apprentissages car il constitue l'espace privilégié de ce développement. Le préscolaire est le lieu d'échanges et de communication et Meirieu (2008 : 8), ne pensait pas pouvoir mieux exprimer le concept de groupe pour dire que : "s'il y a quelque chose que toute l'histoire de la pédagogie nous enseigne...c'est qu'il n'y a pas d'apprentissage cognitif sans rituels de vie collective... ». Avec les autres enfants de son âge, l'enfant de la maternelle est placé dans une situation où la communication est nécessaire. Pendant les activités organisées par l'enseignante, il se construit des échanges verbaux pour aboutir à l'action.

La langue est un outil et un moyen indispensable pour les acquisitions au cours préparatoire. L’acquisition à la maternelle du français langue d'enseignement permet à l'enfant d'avoir une autonomie verbale qui lui permet de rendre compte, au cours des multiples situations, de ses expériences. Les travaux de Caille et Rosenwald (2006) fournissent des résultats comparés d'enfants en situation de maternelle. Ils se rendent compte que les enfants entrés en maternelle à deux ans, présentent bien en moyenne, des performances supérieures à ceux dont la scolarité a débuté à trois ans.

Zazzo (1987), insiste à son tour, sur la participation verbale en grande section de maternelle qui est pour lui, le meilleur prédicteur de l'adaptation scolaire à l'école primaire l'année suivante. Dans le même sens, Florin (1991) a mis en évidence d’importantes transformations de la structure psychologique des élèves entre la première année de scolarisation et la classe de CE1. Dans cette structure, psychologique, la compétence langagière et le niveau de participation aux conversations scolaires jouent un rôle particulièrement important en maternelle pour prédire la réussite scolaire mais en interaction avec d'autres dimensions psychologiques caractérisant l'attitude des enfants face aux tâches scolaires (attention, suivi du rythme de la classe, autonomie, rapidité et efficacité dans les l'exécution d'une tâche...). Ces dimensions vont voir leur poids augmenter à l'école primaire. 
La préscolarisation est l'étape de l'éducation scolaire qui précède l'entrée dans les cours préparatoires de l'enfant. Celui-ci est soumis à un préapprentissage c'est-à-dire, une période de mise en condition qui se situe avant les apprentissages proprement dits. Pendant cette période, l'enfant va s'exercer à perfectionner et forger ses sens, outils d'observation. Régulièrement, des textes lui sont lus, il apprend à écrire des mots plus fréquents comme (le, la, et, un, des, du, les, ma, tes, ta, mon, sa, ses, son, mes, etc.) L'étape de la maternelle permet à l'enfant de reconnaître au moins une quinzaine de lettres dans l'ensemble de l'alphabet et il parvient généralement à les utiliser pour épeler des mots ; il sait également analyser certains mots en syllabes. L'enfant comprend que ce sont les syllabes qu'on doit représenter en écrivant les mots.

L'éducation préscolaire constitue un outil indispensable de formation de l'enfant, le préparant en principe au cycle primaire où il doit pouvoir lire et écrire seul. Cependant, la préscolarisation n’est pas un remède universel à la maîtrise des apprentissages scolaires chez tous les enfants. Le passage de l'enfant à la maternelle ne lui garantit pas nécessairement un succès évident pour ses années primaires comme en témoignent les résultats dans les tableaux. (Lentin 1977 :27) va dans le même sens pour dire que «le rôle de la maternelle est illusoire car il s'appuie sur un modèle d'enfant socialement situé, auquel on s'efforce de conformer tous les sujets quelles que soient entre eux leurs différences objectives, individuelles ou sociales, biologiquement déterminées ou acquises dans le milieu de vie et les conditions déterminées ».

\section{Maturité et développement de l'écriture}

Certains enfants peuvent réussir leur cycle primaire sans préalablement avoir fait la maternelle. C'est le cas des enfants issus des zones rurales où l'école maternelle n'existe pas et qui sont pourtant très appliqués en écriture. Mais l'analyse des résultats d'ensemble de cette étude montre sans équivoque que la fonction écriture constitue pour les apprenants, une activité difficile. La difficulté de l'écriture s'explique en partie par l'immaturité physique chez les enfants des zones urbaines notamment, immaturité caractérisée par une instabilité dans l'exécution des tâches, dans les différents jeux, la fragilité du corps et la maladresse des gestes. Aussi, allant dans le même sens, Delorme (2005: 25) soutient que "pour bien apprendre il faut que l'enfant ait un développement psychomoteur correct. L'écriture est un geste extrêmement complexe puisqu'il va partir de l'épaule au bras, à l'avant-bras, au poignet, à la main pour arriver aux doigts. L'enfant ne peut y parvenir que si toutes ces étapes sont bien franchies dans son enfance. Ce développement psychomoteur n'est pas seulement dans le 
mouvement c'est un ensemble d'interactions complexes entre le moteur et le psychique. C'est l'action qui aide l'intelligence à se construire ».

Saint Marc (1981), résume la difficulté de l'acte graphique pour dire que l'écriture se déploie dans un espace restreint conventionnel (cahier, feuille, ardoise) où le geste lui-même inscrit un mouvement étroit tout en contrôle (place de l'épaule, du coude, du poignet) et en ajustement fin. La contrainte motrice est-elle même dépendante de l'évolution psychologique dont il faut rappeler qu'à 6 ans elle ne place pas tous les écoliers sur un plan d'égales possibilités. L'évolution normale de la motricité enfantine, en particulier, fait que les capacités graphiques des jeunes garçons sont en retard d'un an environ sur celles des filles.

L'écriture repose sur des coordinations et des différentiations de gestes réglés par des sensations de mouvement de posture. Aussi, soutient (Touyarot 1971 :26,) « Certaines maladresses de l'enfant de six ans qui constituent un handicap scolaire considérable, ont pour origine lointaine une coordination insuffisante des tous premiers mouvements et une organisation défectueuse de l'espace et du temps ».

Réaliser la forme d'un graphème, nécessite que l'enfant effectue des gestes naturels, spontanés qui ne demandent pas un apprentissage particulier. Ainsi, l'apprentissage de l'écriture est-il dépendant d'apprentissages antérieurs et plus largement de la façon dont l'enfant a commencé à organiser son univers à partir de ses activités corporelles depuis la cellule familiale. A ce niveau, les enfants des zones rurales sont privilégiés et les résultats de l'étude nous permettent de dire qu'ils réussissent mieux l'écriture que les autres. Quel est ce privilège qui donne aux enfants des zones rurales une longueur d'avance en écriture sur les autres enfants qui ont pourtant fait l'école maternelle. Les enfants des zones rurales présentent de meilleure graphies, parce que comme le dit Amigues et Zerbato, (2000), l'apprentissage de l'écriture n’est pas une activité individuelle mais un véritable processus social qui va de la construction collective (par une confrontation dans un petit groupe d'enfants de procédure d'exécution et des résultats de l'action) à l'appropriation individuelle.

Une telle conception est en phase avec les réalités du milieu culturel dans lequel sont intégrés les enfants et correspondent aux pratiques sociales qui caractérisent leur mode de vie. Le mode de vie en campagne satisfait pleinement à certaines conditions d'entrée dans l'écriture et d'après Amigues et Zerbato (2010), pour maîtriser cette fonction du langage, il convient d'établir des liens sociaux entre l'enfant et l'écrit, insérer les activités et les savoirs dans un projet. Apprendre à écrire est certes fondamental pour l'enfant mais avant, il lui faut intégrer dans ses schèmes cognitifs, des habitudes qu'incarne sa culture et son environnement immédiat. Cela n’est réalisable que sur la base d’une certaine maturité biologique. 
En effet, dans les zones rurales, la scolarisation des enfants en CP1 se fait à partir de huit ans et plus. Le manque de moyens financiers est régulièrement évoqué pour retarder la scolarisation de l'enfant jusqu'à neuf ans en général. Mais avant son entrée à l’école moderne, l’enfant a déjà développé des pratiques quotidiennes en interaction avec le corps social. Ainsi, depuis l'âge de cinq ans, il apprend à tisser des nasses et des paniers qui exigent une certaine coordination des gestes propres à l'exécution de certaines lettres de l'alphabet. L'usage des lance-pierre pour la chasse aux oiseaux par les jeunes garçons, est une anticipation au développement de la musculature chez l'enfant. La petite fille quant à elle, apprend à faire la cuisine. Elle est invitée à piler du foutou ${ }^{14}$ ou faire du kabato ${ }^{15}$ ou même à se tresser les cheveux entre amies.

Ces activités-là, affinent les mouvements de verticalité, de translation et de rotation des mains, chez la jeune fille et comme le soutient ZerbatoPoudou (2014): "Pour écrire en cursive, deux mouvements doivent se coordonner, le mouvement de translation (la main avance de gauche à droite pour écrire le mot) et le mouvement de rotation (les doigts, le poignet, sont mis à contribution pour tracer la forme de chacune des lettres). La coordination entre ces deux types de mouvement se réalisant grâce à l'appui que constitue l'avant-bras, met en jeu une spécialisation des segments du bras, de l'épaule, de l'avant-bras, du poignet, de la main et des doigts. Ainsi, l'écriture cursive, nécessite l'habileté et l'autonomisation des articulations des phalanges et du poignet, chose qui n'est possible qu'entre 5 et 6 ans ». L'enfant du village qui entre en Cp1 à huit ou neuf ans, a suffisamment développé sa psychomotricité. Il capitalise les activités manuelles effectuées en termes d'adresse, généralement bien assimilées, favorisant ainsi son développement psychomoteur qui rend possible, plus tard, une certaine application lors de l'apprentissage de l'écriture. Les habiletés sont en effet développées chez l'enfant par la constance des pratiques manuelles et abordant dans le même sens, Tresse (2014), souligne que «chaque fois que nous apprenons quelque chose de nouveau, les circuits nerveux se modifient. Ainsi, plus on fait travailler certaines connexions plus elles sont performante ». Le jeune enfant a appris dès l’âge de cinq ans, à développer une certaine dextérité par l'usage des travaux manuels en lien avec le développement des schèmes cognitifs qui intègre ses habiletés. Les performances graphologiques qu'il développe à l'école correspondent à bien d’égards, à des antécédents culturels. Cette expérience a sans doute conduit Rogoff (1990) à indiquer que dans les sociétés traditionnelles, les interactions enfants-adultes sont moins verbales mais davantage

\footnotetext{
${ }^{14}$ Foutou : Repas africain fait à partir de banane et de manioc pilés.

${ }^{15}$ Kabato : Repas africain fait à base de farine de mil ou de maïs.
} 
contextualisées, leur objectif étant de parvenir à une intégration réussie. La médiation humaine, une caractéristique typique à la société traditionnelle est déployée par le corps social pour coordonner et ajuster les gestes de l'enfant à travers plusieurs activités. Dans les sociétés modernes au contraire, les interactions sont davantage verbales et moins abstraites dans ce sens qu'elles favorisent le développement chez l'enfant, de compétences qui n’ont pas de valeur pratique immédiate.

\section{Conclusion}

La préscolaire constitue le premier maillon du système éducatif ivoirien. Le passage à l'école maternelle avant d'aborder les apprentissages des cours préparatoires de l'école primaire devient obligatoire. Malheureusement force est de constater que le phénomène de préscolarisation reste essentiellement urbain. Les enfants des zones rurales sont privés de maternelle et pourtant, ils apprennent au même titre que ceux des zones urbaines, les fondements de la langue d'enseignement dans ses fonctions orales et écrites.

A travers cette étude, nous avons pu nous rendre compte que la préscolarisation constitue une variable déterminante dans le développement du langage oral chez les enfants et ce sont ceux des zones urbaines qui en bénéficient largement. Chez les autres enfants qui n’ont pas la facilité d'exprimer leur expérience de vie à travers le français, nous avons pu observer que l'absence d'une faculté en appelle une autre. En effet, chez ces derniers, on remarque la facilité qu'ils ont à former des lettres, à écrire des mots avec une habileté impressionnante.

L'adresse qu'ils ont à manipuler le stylo à bille, fait d'eux, de très bons scripteurs et leur confère la qualité de bons élèves en écriture. Il faut sans doute comprendre que la lecture et l'écriture ne procèdent pas de la même démarche et l'écriture, discipline plus difficile, demande des critères que l'école maternelle ne peut pas satisfaire seule. L’éducation traditionnelle $\mathrm{a}$, à travers ses pratiques sociales, une très grande influence sur le développement de la motricité chez l’enfant.

\section{References:}

1. AMIGUES, R. \& ZERBATO, M-T. (2000), Comment l'enfant devient élève. Les apprentissages à l'école maternelle. Paris : Retz/ HER.

2. AMIGUES, R. et ZERBATO, M-T. (2010). Comment l'enfant devient élève. Les apprentissages à l'école maternelle. Pdf repéré à www.pedacoach.org le 14 Mars 2016. 
3. BAUTIER, E. (2006). Apprendre à l'école, Apprendre l'école. Les risques de construction d'inégalités dès la maternelle. Équipe ESCOL. Lyon. Chronique Sociale.

4. BERNICOT, J. (2009). L'acquisition du langage par l'enfant. Paris. Dans Press.

5. BRUNER, J. (1983). Le développement de l'enfant: savoir-faire, savoir dire. Paris. Puf.

6. CAILLE, J-P. \& Rosenwald, F. (2006). Les inégalités de réussite à l'école élémentaire : Construction et évolution in INSEE (dir). France portrait social. : Paris. Institut National de la Statistique et des Études Économiques. p 115-137.

7. DELORME, J. (2005). L'enfant précoce et les difficultés d'écriture. Repéré à www.afep-asso.fr . Ouvert le 06 Aout 2016.

8. FLORIN, A. (1991). Pratique du langage à l'école maternelle et prédiction scolaire. Paris. Puf.

9. LENTIN, L. (1977). Apprendre à parler à l'enfant de moins de six ans où ? Quand ? Comment ? Paris. ESF.

10. MEIRIEU, P. (2008). Ecole maternelle, école première. Conférence lors du congrès de l'AGEEM. Pdf. Repéré à www.meirieu.com . Ouvert le 17 Mars 2015. P. 9.

11. PASSERIEUX, C. (2009). La maternelle : première école, premiers apprentissages. Lyon. Chronique sociale.

12. PIAGET, J. (2006). La fonction du jeu symbolique. Repéré à www.passerelles-eje.info. Ouvert le 18 Mars 2015.

13. SAINT M, C. (1981). Problèmes et motivation de réalisation. Paris. Didier.

14. SAUVAGE, J. (2003). L'enfant et le langage une approche dynamique et développementale. Paris. L'harmattan.

15. TRESSE, J. (2014). Les avantages du bilinguisme. Repéré dans www.educavox.fr Ouvert le 12 juillet 2016.

16. TOUYAROT, C. (1971). Lecture et conquête de la langue. Paris. Nathan.

17. VYGOTSKI, L. S. (1985). La méthode instrumentale en psychologie. Dans B Schneuly et J.P Bronckart (dir), Vygotski aujourd'hui. (p.3947). Neuchâtel. Delachaux et Niestlé.

18. ZAZZO, B. (1987). Un grand passage de l'école maternelle à l'école élémentaire. Paris. Puf.

19. ZERBATO-P, M, T., (2014). Maternelle: A propos de l'apprentissage de l'écriture. Repéré dans www.cafepedagogique.net le 18 Juillet 2016. 